\title{
Opening the terahertz window on the OSIRIS spectrometer
}

\author{
F. Demmel ${ }^{1, \text { a }}$, D. McPhail ${ }^{1}$, J. Crawford ${ }^{1}$, D. Maxwell ${ }^{1}$, K. Pokhilchuk ${ }^{1}$, V. Garcia-Sakai ${ }^{1}$, S. Mukhopadyay ${ }^{1}$, \\ M.T.F. Telling ${ }^{1}$, F.J. Bermejo ${ }^{2}$, N.T. Skipper ${ }^{3}$ and F. Fernandez-Alonso ${ }^{1,3, b}$ \\ ${ }^{1}$ SIS Facility, Rutherford Appleton Laboratory, Chilton, Didcot OX11 0QX, UK \\ ${ }^{2}$ Instituto de Estructura de la Materia IEM-CSIC, Consejo Superior de Investigaciones Científicas, Serrano 123, Madrid \\ 28006, Spain \\ ${ }^{3}$ Department of Physics and Astronomy, University College London, Gower Street, London WC1E 6BT, UK
}

\begin{abstract}
A cooled and mechanically retractable beryllium filter has been installed and commissioned on the low-energy OSIRIS spectrometer at ISIS. This instrument development extends the energy-transfer range of the spectrometer up to ca. $20 \mathrm{meV}(\sim 5 \mathrm{THz})$, leading to an excellent resolution at $\mathrm{THz}$ frequencies and substantial gains in detected flux relative to existing capabilities on the neighbouring IRIS spectrometer. Herein, we provide a concise account of this new capability for highresolution neutron spectroscopy in the $\mathrm{THz}$ domain, as well as outline a number of ongoing and potential scientific opportunities in condensed-matter physics, chemistry, and materials science.
\end{abstract}

\section{The reasons why}

OSIRIS is an indirect-geometry neutron spectrometer with long-wavelength diffraction capabilities [1]. The secondary spectrometer sits at ca. $34 \mathrm{~m}$ from a $25 \mathrm{~K}$ liquid-hydrogen $\left(\mathrm{H}_{2}\right)$ moderator on ISIS Target Station I. Optimised for low-energy, high-resolution inelastic neutron-scattering studies, OSIRIS uses a broad band of incident wavelengths which are Bragg-scattered from a crystal-analyser array following interaction with the sample under investigation. Owing to the pulsed nature of the source, time-of-flight methods are used to determine energy transfer at a particular scattering angle.

As the last of first-generation instruments built at ISIS, OSIRIS has been operational since 1997. A schematic diagram of the instrument is shown in Fig. 1. In the early days, its primary use was as a long- $d$ spacing diffractometer, exploiting its dedicated, 960-pixel backscattering detector bank with a resolution as high as $\Delta d / d=2.5 \times 10^{-3}$ and a dynamic range extending up to $20 \AA$. In 2003, the instrument was upgraded with a cooled pyrolytic graphite (PG) analyser bank, thereby paving the way for low-energy inelastic and quasielastic neutron-scattering studies [3]. Its primary spectrometer profited from early advances in neutronguide technology in the 1990s, with an $m=2$ guide extending across the majority of its primary length, and terminated by an $m=3.5$ focusing section immediately prior to the sample. In spectroscopic mode, OSIRIS uses PG crystals cooled to $10 \mathrm{~K}$ so as to define two primary final energies using PG002 $\left(\mathrm{E}_{\mathrm{f}}=1.84 \mathrm{meV}\right)$ and PG004 $\left(\mathrm{E}_{\mathrm{f}}=7.38 \mathrm{meV}\right)$ Bragg reflections. The spectral resolution

\footnotetext{
a Corresponding author: F. Demmel (franz.demmel@stfc. ac.uk)

b and F. Fernandwz-Alonso (felix.fernandez-alonso@stf c. ac.uk)
}

of these two alternative settings amounts to 25 and $99 \mu \mathrm{eV}$ for PG002 and PG004, respectively. Recent Monte Carlo simulations of the instrument have also provided important insights into its neutronic performance, including the various contributions to the resolution function and line shape [2]. As on similar neutron spectrometers like IRIS at ISIS [4] or, more recently, BASIS at SNS [5] or DNA at JPARC [6], a tight spectral resolution in the $\mu \mathrm{eV}$ domain comes as a result of several factors, including: a relatively long flight path $(>30 \mathrm{~m})$ at a short-pulse spallation source; tight incident neutron pulses from a cold neutron moderator (which, as in the case of DNA, it may require the additional use of a pulse-shaping chopper in the primary spectrometer); a low-energy (few meV) analyser for spectral analysis as afforded by materials like PG or silicon; and an analyser geometry close to back-scattering $\left(>85^{\circ}\right)$.

In addition to simultaneous high-resolution diffraction capabilities, the high spectral resolution and wide dynamic range afforded by this family of inelastic neutronscattering instruments provide a number of opportunities for low-energy spectroscopy. To illustrate the above, Fig. 2 shows inelastic neutron-scattering spectra centred at the $0 \rightarrow 1$ rotational transition of the $\mathrm{H}_{2}$ molecule at ca. $15 \mathrm{meV}$. On a steady reactor source, this spectral feature is readily accessible using thermal incident wavelengths, leading to a spectral resolution around $1 \mathrm{meV}$ [7]. As shown in the figure, an improvement by a factor of around three may be attained on broadband crystalanalyser spectrometers like TOSCA at ISIS [8]. IRIS has already demonstrated a much tighter spectral response, as shown by a resolution-limited feature of ca. $100 \mu \mathrm{eV}$ at $15 \mathrm{meV}$ in the same figure [9]. At present, going beyond these capabilities requires the use of inelastic-spin-echo techniques, well within the $\mu \mathrm{eV}$ domain as illustrated in Fig. 2 and Ref. [7]. The bottom panel of Fig. 2 also

This is an Open Access article distributed under the terms of the Creative Commons Attribution License 4.0, which permits unrestricted use, distribution, and reproduction in any medium, provided the original work is properly cited. 


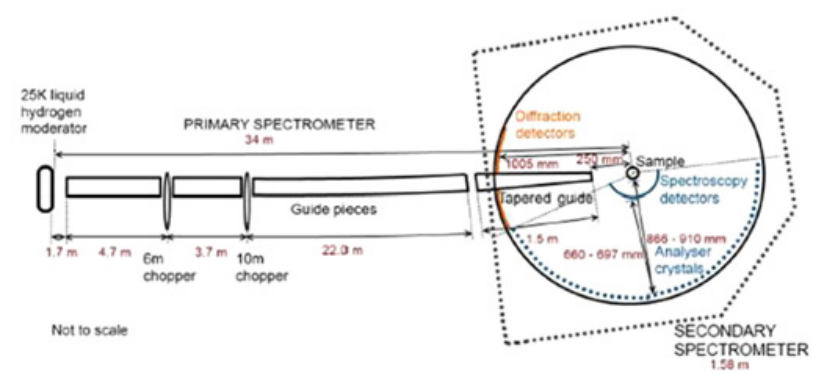

Figure 1. Schematic diagram of the OSIRIS spectrometer. For further details, see the main text and Ref. [2].
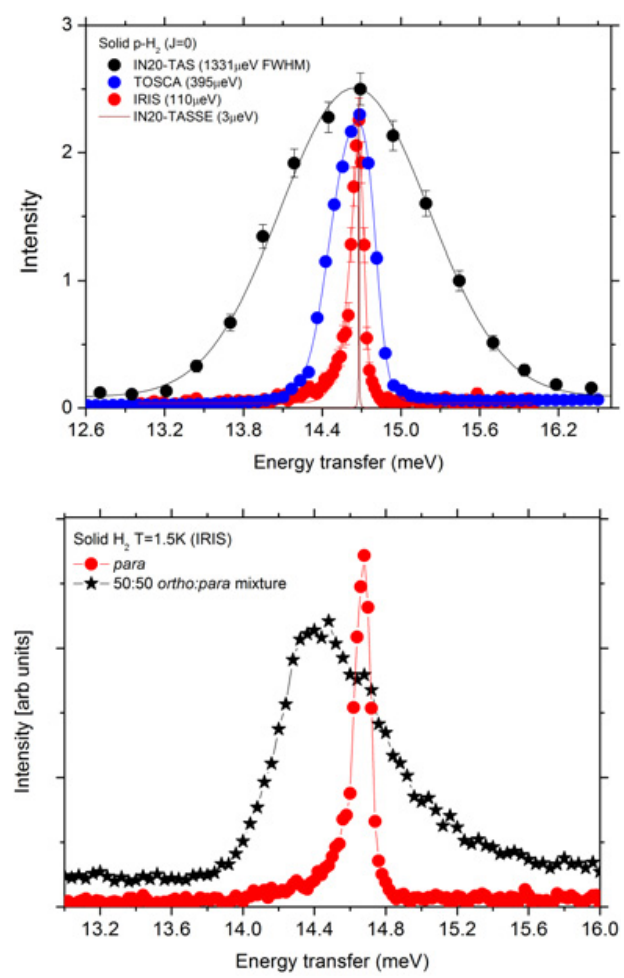

Figure 2. Top: inelastic neutron-scattering spectra of the first rotational line of bulk $p-\mathrm{H}_{2}$. Bottom: rotational spectra of solid $p-\mathrm{H}_{2}$ in the absence (red dots) and presence of $o-\mathrm{H}_{2}$ (black dots). In both cases, positive energy transfer denotes neutron-energy loss. For further details, see the main text.

illustrates the additional information content associated with the use of a near-backscattering spectrometer like IRIS or OSIRIS for inelastic studies. In the particular case of $p-\mathrm{H}_{2}$ in its ground rotational level, interactions with $o$ $\mathrm{H}_{2}(\mathrm{~J}=1)$ molecules lead to an overall redshift and distinct broadening of this transition, phenomena associated with subtle (yet at the same time observable) intermolecular interactions in the solid hydrogens.

On an instrument like IRIS, clean access to energy transfers up to $15-20 \mathrm{meV}$ has been possible owing to the use of a beryllium $(\mathrm{Be})$ filter in the secondary spectrometer to suppress Bragg reflections beyond the lowest order, namely, PG002. Although this rather beneficial feature has been available on IRIS for some time now, high-resolution inelastic-neutron scattering studies well beyond $\sim 1 \mathrm{THz}$ $(1 \mathrm{meV}=0.24180 \mathrm{THz})$ have been severely limited by
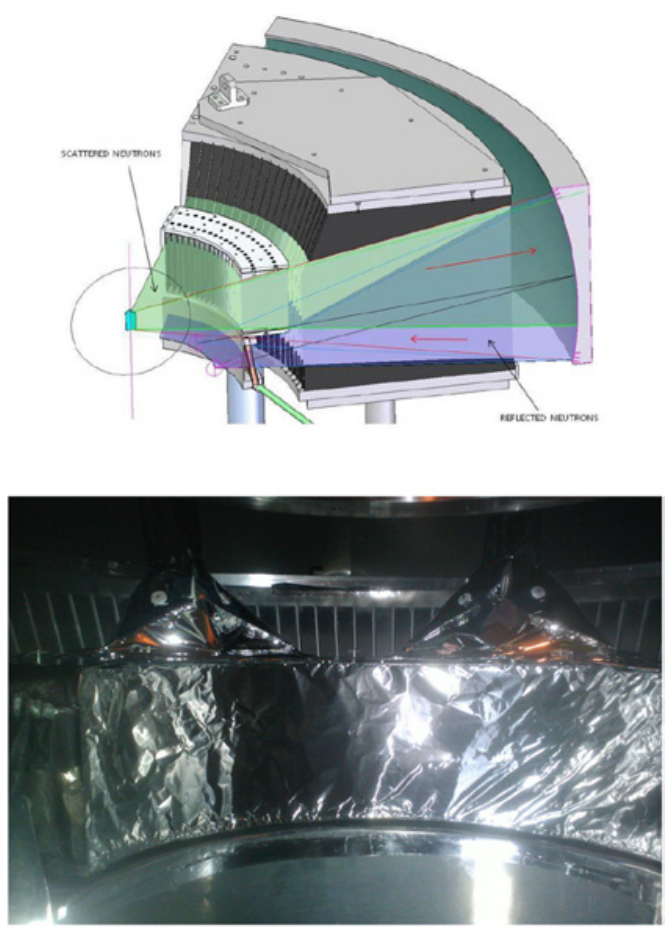

Figure 3. Top: schematic drawing of the OSIRIS secondary spectrometer highlighting in green the neutron paths through the Be filter after scattering by the sample. Bottom: photograph of the actual Be-filter assembly inside the OSIRIS tank, showing the radial arrangement of the $\mathrm{Be}$ wedges as described in the main text. The Be blocks are enclosed within a thermal shield and additional multilayer insulation. Two copper legs link the top of the filter assembly to closed-cycled refrigerator (CCR) heads located outside the vacuum tank. In this photograph, the radial collimator can be seen behind the filter.

intrinsic flux limitations on this day-one ISIS instrument. Reasons include the use of a natural-nickel guide on the primary spectrometer, as well as a relatively small PG analyser bank.

To circumvent the above limitations, a new Be filter has been designed, constructed, and installed on the neighbouring OSIRIS spectrometer. In this contribution, we summarise its main technical characteristics and performance.

\section{The new beryllium filter}

In the new Be filter on OSIRIS, scattered neutrons from the sample are transmitted through wedged, polycrystalline Be blocks, leading to the suppression of PG00X $(X>2)$ reflections prior to neutron reflection on the $\mathrm{PG}$ analyser, and subsequent detection using an array of forty-two ${ }^{3} \mathrm{He}$ tubes.

As shown in more detail in Fig. 3, the filter has the same angular mapping as that of the radial collimator on the instrument, with a total of forty-two independent $\mathrm{Be}$ slats decoupled by $1 \mathrm{~mm}$ sheets of cadmium $(\mathrm{Cd})$. The design criteria for the OSIRIS Be filter have capitalised from prior experience with similar devices on IRIS and TOSCA at ISIS. Given a total cross section for Be of 7.63 barn and a particle density of $1.24 \times 10^{23} \mathrm{~cm}^{-3}$ [10], 
low-energy neutrons have a mean-free path of ca. $1 \mathrm{~cm}$ in the material. As this cross section is dominated by coherent scattering within the crystalline lattice above an energy cutoff of ca. $5 \mathrm{meV}$, a suitable absorbing material (in this case $\mathrm{Cd}$ ) is required to minimise detrimental secondary backgrounds reaching other elements within the instrument and, ultimately, the detector array. In our particular case, this condition has been met by surrounding each individual $\mathrm{Be}$ slat with thin $\mathrm{Cd}$ sheets of the same length and height as the former. The overall length of the Be slats were chosen so as to be in excess of ten neutron mean-free paths in Be, a limit that could not be surpassed owing to spatial constraints on the instrument.

The filter has been placed before the radial collimator and analyser bank to minimise direct line-of-sight by the neutron-detector array, which remains positioned immediately below the filter when operational. In addition, the presence of the radial collimator between sample and PG analyser also serves to reduce the levels of unwanted neutrons reaching the detector bank. To increase the transmission of low-energy neutrons through $\mathrm{Be}$, the filter has to be cooled [11]. Two independent CCRs enable the cooling of the entire unit to temperatures below $40 \mathrm{~K}$, as measured by a set of 8 independent sensors directly attached to the filter assembly. The CCR heads are mounted outside the vacuum tank and are connected to the filter through copper legs. A motorised, computer-driven stage can bring the filter into position for PG002 operation, or out of position if the lowerenergy-resolution PG004 option is required to access a wider momentum-transfer range. The filter is retractable in vacuo, thereby enabling changes in spectroscopic settings on the instrument without further intervention.

\section{Performance}

The performance of the new OSIRIS Be filter has been assessed using a cylindrical vanadium (V) standard at room temperature. The recorded intensity at elastic energy transfers with the filter in place (hereafter "filter-in") is reduced by about $40 \%$ compared to the "filter-out" measurement. This reduction in intensity is the result of a number of factors, including: the overall transmission of $\mathrm{Be}$, the presence of $\mathrm{Cd}$ sheets between the Be wedges, and a reduced angular acceptance of the PG array in the vertical direction in the filter-in position. The latter one arises from design constraints on the instrument which limit the length of the wedges as well as the lowest-possible position of the filter to avoid direct contact with the neutron-detector array. Figure 4 shows angle-averaged inelastic neutron data with and without the filter obtained at $16 \mathrm{~Hz}$ (one third of the repetition rate of ISIS Target Station I).

These data demonstrate the suppression of both PG004 and PG006 reflections with the filter, thereby enlarging the energy-transfer window of the instrument to ca. $20 \mathrm{meV}(\sim 5 \mathrm{THz})$. The new OSIRIS Be filter therefore offers comparable transmission characteristics as those on IRIS. The inset in Fig. 4 shows the suppression of the PG004 reflection in more detail. From integrated intensities, one can deduce that $99 \%$ of PG004 neutrons are suppressed. The remaining fraction arises from mechanical

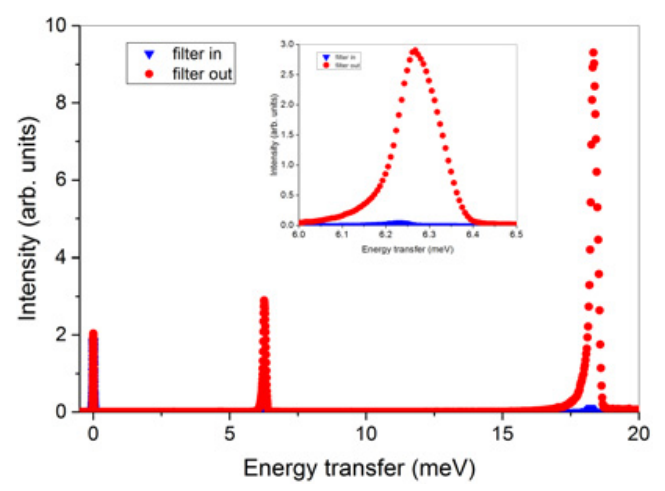

Figure 4. Angle-averaged V spectra on OSIRIS, demonstrating the suppression of higher-order PG00X (X > 2) Bragg reflections with the filter-in configuration. For clarity, the inset shows these data around the 004 reflection.

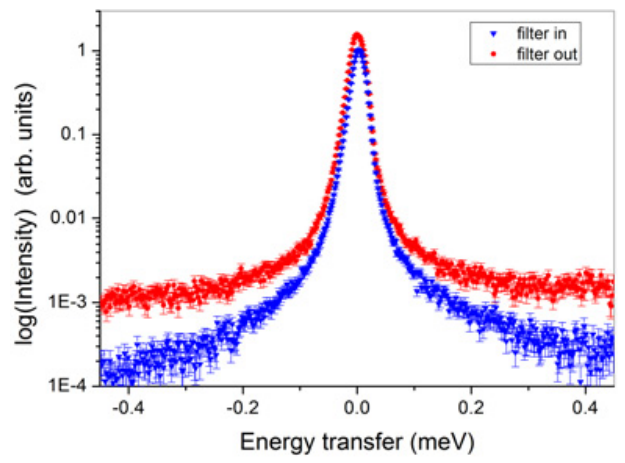

Figure 5. V spectra with and without filter (PG002 reflection). The logarithmic intensity scale serves to demonstrate a sensible increase in signal-to-background ratios in the quasielastic region using the new filter.

imperfections in the filter assembly, in particular between Be wedges and $\mathrm{Cd}$ sheets.

A closer look at the quasielastic region around the PG002 Bragg reflection is presented in Fig. 5. These data show that the filter leads to a reduction of background levels by a factor of 4-5. Taking into account the concomitant reduction in detected flux for the filter-in setting, the new OSIRIS Be filter therefore leads to a net improvement in signal-to-background ratios of a factor of 3 in this energy-transfer range relative to IRIS.

In parallel with the above developments, extensive neutron-transport simulations of the OSIRIS primary and secondary spectrometers have been performed using the McStas code [2]. These simulations provide a necessary starting point to assess in detail the new spectroscopic capabilities of the instrument, as summarised in Fig. 6. For the PG002 reflection, OSIRIS has a slightly relaxed resolution relative to IRIS around the elastic line $(25 \mu \mathrm{eV}$ vs. $17.5 \mu \mathrm{eV})$. These differences become less significant at higher energy transfers, where the contribution from the primary spectrometer to the total spectral resolution (virtually identical in both cases) dominates. The predictions shown in Fig. 6 are not only consistent with previous results on IRIS but at the same time evince a superb spectral resolution up to $20 \mathrm{meV}$ in neutronenergy loss. On OSIRIS, this excellent energy resolution 


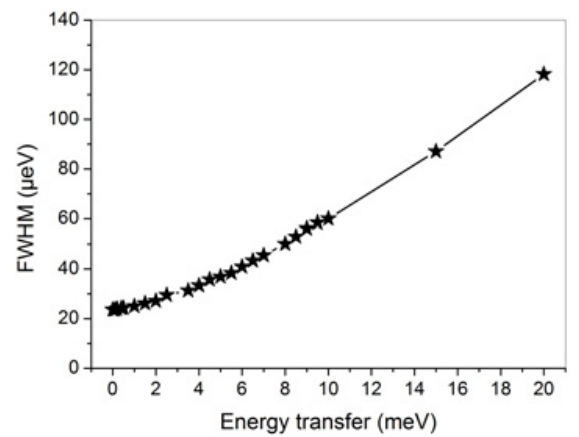

Figure 6. Energy resolution of OSIRIS as a function of energy transfer obtained from Monte Carlo simulations. For further details, see the text.

is accompanied by a much higher flux at these high energy transfers compared to IRIS owing to the use of an $m=2$ supermirror guide in the primary spectrometer. Higher $m$ numbers could also be used in the future to enhance these capabilities further.

\section{Outlook}

The new Be filter on OSIRIS opens up new possibilities in quasielastic studies due to a higher sensitivity of the spectrometer around the elastic line. Furthermore, this instrument development provides new opportunities in high-resolution inelastic spectroscopy with access to spectral features up to $20 \mathrm{meV}$ combined with a high flux. Recent examples which have already explored the lower end of this spectral window include the investigation of excitation lifetimes and line shapes in $\mathrm{CeCoIn}_{5}$ [12] and in the gapped quantum magnet copper nitrate [13]. Further developments in the horizon on the instrument include achieving a tighter spectral resolution. This objective could be readily achieved with a second silicon analyser bank, to be housed on the vacant side of the spectrometer.

The authors gratefully acknowledge the UK Science \& Technology Facilities Council for financial support and access to beam time at ISIS.

\section{References}

[1] www.isis.stfc.ac.uk/instruments/osiris/ (last accessed 22 September 2014)

[2] K. Pokhilchuk, Rutherford Appleton Laboratory Technical Report RAL-TR-2013-008 (Chilton, 2013, ISSN 1358-6254). Report available at: epubs.stfc.ac.uk/work/65985 (last accessed 22 September 2014)

[3] M.T.F. Telling and K.H. Andersen, Phys. Chem. Chem. Phys. 7, 1255 (2005)

[4] C.J. Carlile and M.A. Adams, Physica B 182, 431 (1992)

[5] E. Mamontov and K.W. Herwig, Rev. Sci. Instrum. 82, 85109 (2011)

[6] M. Arai et al, J Phys Soc Jpn 82 SA024 (2013)

[7] F. Fernandez-Alonso et al., Phys. Rev. B 86, 144524 (2012)

[8] wwwisis2.isis.rl.ac.uk/INSdatabase/ (last accessed 22 September 2014)

[9] F. Fernandez-Alonso et al., Phys. Rev. Lett. 98, 215503 (2007)

[10] J. Dawidowski et al., in Neutron Scattering Fundamentals, F. Fernandez-Alonso and D.L. Price eds. (Academic Press, New York, 2013), Appendix

[11] A.K. Freund, Nucl. Instr. Meth. 213, 495 (1983)

[12] C. Stock et al., Phys. Rev. Lett. 109, 167207 (2012)

[13] D.A. Tennant et al., Phys. Rev. B 85, 014402 (2012) 